Research Article

\title{
The Dynamics of a Spatial Economic Model with Bounded Population Growth
}

\author{
Yue Zhong $\mathbb{D}$ \\ School of Science, Xihua University, Chengdu 610039, China \\ Correspondence should be addressed to Yue Zhong; zhongyue@smail.swufe.edu.cn
}

Received 2 April 2021; Revised 14 July 2021; Accepted 26 July 2021; Published 5 August 2021

Academic Editor: Chris Goodrich

Copyright $\odot 2021$ Yue Zhong. This is an open access article distributed under the Creative Commons Attribution License, which permits unrestricted use, distribution, and reproduction in any medium, provided the original work is properly cited.

\begin{abstract}
We investigate a spatial economic growth model with bounded population growth to obtain the asymptotic behavior of detrended capital in a continuous space. The formation of capital accumulation is expressed by a partial differential equation with corresponding boundary conditions. The capital accumulation interacts with the morphology to affect the optimal dynamics of economic growth. After redrafting the spatial growth model in the infinite dimensional Hilbert space, we identify the unique optimal control and value function when the bounded population growth is considered. With nonnegative initial distribution of capital, the explicit solution of the model is obtained. The time behavior of the explicit solution guarantees the convergence issue of the detrended capital level across space and time.
\end{abstract}

\section{Introduction}

Distribution of economic activities across space and time has been widely investigated in many literature [1-5], where economic geographers apply economic growth models to analyze the relationship between production agglomeration and location choices of people, reasons for migration flows, and formation of cities. In early studies of regional economic growth models, Beckmann [6] and Isard and Liossatos [7] discussed flows of labor and pollution, capital accumulation, and individuals welfare in discrete and continuous spaces, respectively. Recently, as one important issue of economic research studies, optimal market allocation has been considered in economic growth models with capital accumulation. Among relevant economic literature, Brito [8] has investigated the optimal dynamics of spatial capital accumulation in a continuous space. In the work of Brito [8], optimal consumption distribution is associated with the capital accumulation, which drives the dynamic processes of spatial capital growth and distribution. Maximizing an objective function in a continuous dimension, Boucekkine et al. [9] illustrated how and why policy makers choose optimal trajectories of the capital and consumption in a spatial Ramsey model. Considering a cyclic space,
Boucekkine et al. [10] described characterizations of the optimal capital dynamics in the spatial Ramsey model with AK production function, which indicates that technology adoption decisions are closely tied to technological embodiment, capital, and goods. In a generic geographic structure, Fabbri [11] generalized the spatial AK economic growth model presented in [10] to obtain a generalized qualitative behavior of the economy.

In global economic growth systems, several factors affect the formation of capital accumulation. These factors include population growth, environmental protection, and different production processes. For example, Guerrini [12] studied the one-dimensional Ramsey model with a population growth factor. The population growth rate is considered to be a variable, which follows the logistic growth law. La Torre et al. [13] discussed the interplay of pollution diffusion and capital accumulation in economic geography models, where the dynamics of spatial capital is driven by the pollution diffusion. In Kamihigashi and Roy [14], a discontinuous production function is applied to show the convergence of the capital stock when an optimal growth problem with the condition of discrete time is considered. Brianzoni et al. [15] considered the optimal dynamics of capital accumulation in a discrete economic model with one sector, in which 
nonconcave production factors lead to the complexity of capital stock in local and global economic systems. Capasso et al. [16] analyzed the relationship between industrial dynamics and economic geography to enrich the theory of spatial economy.

In general, it is very hard to obtain the explicit solutions of spatial economic growth systems. Thus, the equilibrium distribution of spatial capital is widely considered in many research studies. Camacho and Zou [17] discussed existence of the solution for a spatial growth system with local diffusion and obtained convergence properties of capital distribution in the long run. Under assumption of a nonconcave production function, Capasso et al. [18] investigated the steady state of a spatial Solow model with technological diffusion. Brock et al. [19] applied a mathematical method to study spatial spillover in an economic growth model, in which capital mobility is excluded since the diffusion term disappears from the state equation. In the long run, Xepapadeas and Yannacopoulos [20] illustrated stable distribution of per capita capital across space. The stable capital distribution is related to the growth process of economy and shows nonhomogeneous properties. In autarkic and open regions, Yue and Wenyi [21] depicted the formation of capital distribution by applying the equilibrium solution of a generalized growth model, respectively. For more contributions to the study of growth models, the reader is referred to [22-29] and the references therein.

In this paper, we introduce a bounded population growth factor into the spatial economic growth model presented in $[10,11]$ and analyze the optimal dynamics of the model in a continuous space. In our model, the optimal behavior of the economic growth is affected by the interaction between the morphology and the dynamics of spatial capital. The long-run convergence of the spatial capital is closely associated with the bounded population growth factor and geographical environment. After redrafting the spatial growth model in the infinite dimensional Hilbert space, we use the Hamilton-Jacobi-Bellman (HJB) equation and a linear production function to derive an explicit value function of the model. The value function is used to confirm the optimal control of the model in feedback form. Due to application of the optimal control, an explicit solution of the growth system is obtained in close form, which satisfies firstorder optimality conditions to describe the time behavior of per capita capital. With nonnegative initial distribution of capital, we apply the explicit solution and Fourier series to express spatial detrended capital of our model. The asymptotic behavior of the spatial detrended capital distribution is proved to show that the capital distribution converges to a homogeneous result in the long run.
In particular, we extend parts of works in $[10,11]$ by embedding the bounded population growth factor into the spatial growth model. In our discussion, the bounded population growth is one determinant of the spatial capital dynamics. When the bounded population growth rate is considered, we identify the unique optimal control and value function, which are used to derive the explicit solution of the model. Compared with the works in $[10,11]$, we apply the explicit solution and Fourier series to obtain an expression of the spatial detrended capital which helps to prove the longrun convergence of the spatial detrended capital. This is the main contribution in our work. In addition to referring the research methods in $[10,11]$, our work is also related to the study of Chatterjee [30], where the optimal control problem of a growth model with heterogeneous initial endowments is solved.

This paper is organized as follows. Section 2 states the growth model with bounded population growth and special spatial setting. In Section 3, the spatio-temporal capital dynamics of the growth model is analyzed by identifying the unique optimal control and the explicit value function. In Section 4, we apply the time behavior of per capita capital in close form to prove convergent properties of the spatial detrended capital distribution. Some numerical results are illustrated in Section 5, and our conclusions are presented in Section 6.

\section{A Spatial Growth Model with Bounded Population Growth}

2.1. A Model with Bounded Population Growth. Let $\Omega$ be an $d$-dimensional geographic structure $(d=2$ or 3$)$ and without boundary. We consider that economic activities are distributed homogeneously in geography $\Omega$. Assume that $x \in \Omega$ is a generic spatial variable and $k(x, t)$ denotes spatial capital stock of the individual located at $x$ and time $t \geq 0$. The population stock $L(x, t)$ is considered to follow a logistic process:

$$
\dot{L}(x, t)=(a-b L(x, t)) L(x, t)
$$

for all $(x, t) \in \Omega \times[0, T]$, where $\dot{L}(x, t)=\partial L(x, t) / \partial t$ and $a>b>0$. The population growth rate is denoted by $N(x, t)=\dot{L}(x, t) / L(x, t)$. Thus, logistic equation (1) implies the upper and lower limits to the population growth rate which satisfies a hypothesis of the bounded population growth rate [12].

In open regions, we ignore intertemporal adjustment costs caused by the flows of capital and goods. When there is no capital depreciation, we have an aggregate balance equation in the geography $\Omega$, which is given by

$$
\int_{\Omega}\left\{\frac{\partial k(x, t)}{\partial t}-A k(x, t)+N(x, t) k(x, t)+i(x, t)+c(x, t)\right\} \mathrm{d} x=0 .
$$

In equation (2), $c(x, t)>0$ denotes the consumption of the individual at point $x$ and time $t$, and $i(x, t) \neq 0$ is the net trade balance of individual at $x$ and time $t$. Note that, in this paper, the production function is considered as the linear 
function ( $A$ is a positive constant). The trade $i(x, t)$ is symmetry of capital account balance that is matched by reallocations of the capital in different regions. In addition, we assume that there is no inter-regional arbitrage opportunities among regions. From equation (2), we obtain the budget constraint which is written as

$$
\frac{\partial k(x, t)}{\partial t}=A k(x, t)-N(x, t) k(x, t)-i(x, t)-c(x, t), \Omega \times[0, T] .
$$

Following the idea in [8], we assume that there are no institutional barriers when capital and goods flow from one region to others. In this case, the net trade balance $i(x, t)$ is determined by different capital intensities among regions. Then, for a region $E$ that is a bounded open subset of $\Omega$, the trade balance $i(x, t)$ satisfies

$$
\int_{E} i(x, t) \mathrm{d} x=-\int_{\partial E} \frac{\partial k i(x, t)}{\partial \widehat{n}} \mathrm{~d} x .
$$

Applying the divergence theorem to equation (4), we get $\int_{\partial E} \frac{\partial k(x, t)}{\partial \widehat{n}} \mathrm{~d} x=\int_{E} \nabla \cdot \nabla k(x, t) \mathrm{d} x=\int_{E} \triangle_{x} k(x, t) \mathrm{d} x$.

Thus, we obtain

$$
i(x, t)=-\triangle_{x} k(x, t) .
$$

Substituting equation (6) into equation (3), we have the following system:

$$
\left\{\begin{array}{l}
\frac{\partial k(x, t)}{\partial t}=\triangle_{x} k(x, t)+A k(x, t)-N(x, t) k(x, t)-c(x, t), \\
(x, t) \in \Omega \times[0, T], \\
k(x, 0)=k_{0}(x)>0, \quad x \in \Omega .
\end{array}\right.
$$

Note that, different from the model presented in $[10,11]$, the population growth factor (the term $N(x, t) k(x, t))$ in system (7) describes the impact of the population growth on capital accumulation.

In order to illustrate the optimal dynamics of economic activities, a control $c(\cdot, \cdot)$ is chosen to maximize utility functional:

$$
J\left(k_{0}, c(\cdot, \cdot)\right):=\int_{0}^{+\infty} e^{-\rho t} \int_{\Omega} \frac{c(x, t)^{1-1 / \sigma}}{1-1 / \sigma} \mathrm{d} x \mathrm{~d} t,
$$

where the constants $\rho>0$ and $\sigma>1$. For a given initial distribution $k_{0}(\cdot)$ of the capital, the value function of system (7) is defined as

$$
V\left(k_{0}\right):=\sup _{c(\cdot, \cdot)} J\left(k_{0}, c(\cdot, \cdot)\right) .
$$

In equation (9), the supremum is associated with a suitable control $c(\cdot, \cdot)$ which ensures that spatial capital is nonnegative at any point $x$ and time $t$.
2.2. The Hilbert Space Setting. For convenience, we state the Hilbert space setting to rewrite the optimal control problem (8). Let $L^{2}(\Omega)$ denote the Hilbert space, in which we describe the dynamics of spatial capital by the adjusted model. In $L^{2}(\Omega)$, the scalar product of $f$ and $g$ is defined as

$$
\langle f, g\rangle=\int_{\Omega} f(x) g(x) \mathrm{d} x,
$$

and the norm of the function is given by

$$
|f|_{L^{2}(\Omega)}:=\langle f, f\rangle^{1 / 2}=\left(\int_{\Omega} f^{2}(x) \mathrm{d} x\right)^{1 / 2} \text {. }
$$

In addition, the distance between two elements $f, g \in L^{2}(\Omega)$ is denoted by $|f-g|_{L^{2}(\Omega)}$. For time $t \in[0, T]$, space variables $k(\cdot, t)$ and $c(\cdot, t)$ are considered as elements of the Hilbert space $L^{2}(\Omega)$. In order to depict the second derivative of functions in $L^{2}(\Omega)$, we express operator $G$ as

$$
G(f)=\triangle_{x} f,
$$

where $G$ is the generator of the heat semigroup, and it is also a $C_{0}$ semigroup on $L^{2}(\Omega)[31,32]$. We denote a subset of $L^{2}(\Omega)$ by $D(G)$ that is the domain of the operator $G . D(G)$ is denoted by the Sobolev space $H^{2}(\Omega)$, which is the completion for the norm $|f|_{H^{2}}:=\left(\sum_{i=0}^{2} \int_{\Omega}\left|\nabla^{i} f(x)\right|^{2} \mathrm{~d} x\right)^{1 / 2}$ in the space of the $C^{\infty}$ functions [31]. Thus, the functions in $D(G)$ have first and second derivatives in the domain $L^{2}(\Omega)$.

Since the population growth rate $N(x, t)$ satisfies the logistic equation allowing the limits to the population growth, for simplifying calculation, we denote the logistic population growth rate $N(x, t)$ by $n$ which is independent of $t$, and we verify the lower and upper limits of the population growth rate in Proposition 1 and Theorem 3, respectively. Based on the previous analysis, in the Hilbert space $L^{2}(\Omega)$, system (7) is rewritten as the following form:

$$
\left\{\begin{array}{l}
\frac{\partial k(t)}{\partial t}=G k(t)+A k(t)-n k(t)-c(t), \quad t \geq 0, \\
k(0)=k_{0}
\end{array}\right.
$$

where $c(t)$ and $k(t)$ are elements of $L^{2}(\Omega)$ for any $t \geq 0$. Note that, $c(t)$ and $k(t)$ are considered as the functions of space variable $x$ and expressed by $c(t)(x)$ and $k(t)(x)$, respectively. That is to say, $c(t)$ and $k(t)$ still denote consumption and capital at time $t$ with space point $x$, respectively $[10,11]$. Therefore, we write $c(t)(x) \equiv c(x, t)$ and $k(t)(x) \equiv k(x, t)$ in the sequel.

We apply the notation $L_{\text {loc }}^{2}\left(L^{2}(\Omega) ;(0,+\infty)\right)$ to denote a function space, which reads

$$
\begin{aligned}
& L_{\mathrm{loc}}^{2}\left(L^{2}(\Omega) ;(0,+\infty)\right) \\
& :=\left\{f: \Omega \times(0,+\infty) \longrightarrow R: \int_{0}^{T} \int_{\Omega}|f(x, t)|^{2} \mathrm{~d} x \mathrm{~d} t<\infty, \quad \forall T>0\right\},
\end{aligned}
$$

and express the set of admissible controls as

$$
\begin{aligned}
& U_{k_{0}}:=\left\{c \in L_{\mathrm{loc}}^{2}\left(L^{2}(\Omega) ;(0,+\infty)\right): c(t)(x), k(t)(x) \geq 0,\right. \\
& \forall(x, t) \in \Omega \times(0,+\infty)\} .
\end{aligned}
$$


Thus, value function (9) is rewritten as

$$
V\left(k_{0}\right)=\sup _{c(\cdot) \in U_{k_{0}}} J\left(k_{0}, c(\cdot)\right) \text {. }
$$

Remark 1. Choosing $c(\cdot) \in U_{k_{0}}$ for an initial capital $k_{0} \in L^{2}(\Omega)$, we know that $k(x, t)$ is the solution of system (7) if the solution of system (13) is denoted by $k(t)$. In addition, it is not necessary to ensure that the initial distribution and the control are regular. In this case, the solution of system (7) is not required to be $C^{2}$ for the space variable and $C^{1}$ for the time variable. Therefore, the unique solution of system (7) is obtained in other form, i. e., it belongs to $C\left(L^{2}(\Omega) ; t(0,+\infty)\right)$ $[32,33]$. We define function $1: \Omega \longrightarrow R$ in $L^{2}(\Omega)$, which is a constant equal to 1 in $\Omega$, that is,

$$
1(x) \equiv 1 .
$$

Thus, utility functional (8) is rewritten as the form

$$
\begin{aligned}
J\left(k_{0}, c(\cdot, \cdot)\right) & :=\int_{0}^{+\infty} e^{-\rho t} \int_{\Omega} \frac{c(x, t)^{1-1 / \sigma}}{1-1 / \sigma} \mathrm{d} x \mathrm{~d} t, \\
& =\int_{0}^{+\infty} e^{-\rho t}\langle 1, U(c(t))\rangle \mathrm{d} t,
\end{aligned}
$$

where function $U(\theta)(x)=\theta(x)^{1-1 / \sigma} / 1-1 / \sigma$ for a given $\theta \in L^{2}(\Omega)$.

\section{Spatio-Temporal Capital Dynamics}

In this section, we use the dynamic programming method to analyze spatio-temporal dynamics of the capital in $L^{2}(\Omega)$. Since optimal control problem (8) is equivalent to maximizing (18) subject to system (13), we concentrate our attentions on HJB equation related to system (13) to discuss the optimal control and explicit value function of system (13). The HJB equation of system (13) is given by

$$
\begin{aligned}
\rho v(k)= & \langle k, G \nabla v(k)\rangle+A\langle k, \nabla v(k)\rangle-n\langle k, \nabla v(k)\rangle \\
& +\sup _{c \in L^{2}\left(T: R^{+}\right)}\{-\langle c, \nabla v(k)\rangle+\langle 1, U(c)\rangle\},
\end{aligned}
$$

where the function $v: L^{2}(\Omega) \longrightarrow R$, i.e., for any $k \in L^{2}(\Omega)$. In equation (19), the term $\langle k, G \nabla v(k)\rangle$ equals $\langle G k, \nabla v(k)\rangle$ since $G$ is self-adjoint. According to the concept of Gateaux derivative, the gradient of the function $v(k)$ (denoted by $\nabla v(k)$ ) is an element of $L^{2}(\Omega)$. Then, the gradient $\nabla v(k)$ at a certain point $x$ is computed in $k \in L^{2}(\Omega)$. Furthermore, since $\nabla v(k) \in L^{2}(\Omega)$, we obtain $G \nabla v(k)$ at the point $x$ when $\nabla v(k)$ is in the domain $D(G)$ [34]. Similar results appear in the standard dimensional case. This fact allows us to expect that the solution $v(k)$ of the HJB equation equals the value function of the infinite dimensional problem.

Let $M:=\left\{k \in L^{2}(\Omega):\langle k, 1\rangle>0\right\}$ be an open set. We consider a continuous function $v \in C^{l}(M)$ with continuous differential, which means that the gradient $\nabla v: M \longrightarrow L^{2}(\Omega)$ is continuous in $L^{2}(\Omega)$. Now, in order to obtain the optimal control of system (13) in feedback form, we identify an explicit solution (denoted by $v$ ) of equation (19) on $M$ in the following proposition.

Proposition 1. On the half space $:=\left\{k \in L^{2}(\Omega):\langle k, 1\rangle>0\right\}$, if the population growth rate $n$ satisfies

$$
n>A+\frac{\sigma \rho}{1-\sigma},
$$

then there exists an explicit solution of HJB equation (19), which is given by

$$
v(k)=\mu\langle k, 1\rangle^{1-1 / \sigma},
$$

where

$$
\mu=\frac{\sigma}{\sigma-1}\left(\frac{\sigma \rho-(A-n)(\sigma-1)}{\operatorname{vol}(\Omega)}\right)^{-1 / \sigma}
$$

for $\operatorname{vol}(\Omega):=\int_{\Omega} 1 d x$.

Proof. Considering $v(k)=\mu\langle k, 1\rangle^{1-1 / \sigma}$ for a positive real number $\mu$, we have

$$
\nabla v(k)=\mu\left(1-\frac{1}{\sigma}\right)\langle k, 1\rangle^{-1 / \sigma} 1 .
$$

Since $\nabla v(k)$ belongs to the domain $D(G)$ and $G \nabla v(k): M \longrightarrow L^{2}(\Omega)$ is continuous for any $k \in M$, then substituting $v(k)=\mu\langle k, 1\rangle^{1-1 / \sigma}$ into equation (19), we obtain

$$
\begin{aligned}
\rho \mu\langle k, 1\rangle^{1-1 / \sigma}= & \mu\left(1-\frac{1}{\sigma}\right)\langle k, 1\rangle^{-1 / \sigma}\langle k, G 1\rangle+A \mu\left(1-\frac{1}{\sigma}\right)\langle k, 1\rangle^{-\frac{1}{\sigma}}\langle k, 1\rangle,-n \mu\left(1-\frac{1}{\sigma}\right)\langle k, 1\rangle^{-1 / \sigma}\langle k, 1\rangle \\
& +\sup _{c \geq 0}\left\{-\mu\left(1-\frac{1}{\sigma}\right)\langle k, 1\rangle-\frac{1}{\sigma}\langle c, 1\rangle+\langle 1, U(c)\rangle\right\} .
\end{aligned}
$$


Due to $G 1=0$, the supremum is obtained if $c=(\mu(1-1 / \sigma))^{-\sigma}\langle k, 1\rangle 1$ in equation (24). Thus, we write equation (24) as

$$
\begin{aligned}
\rho \mu\langle k, 1\rangle^{1-1 / \sigma}= & A \mu\left(1-\frac{1}{\sigma}\right)\langle k, 1\rangle^{-1 / \sigma}\langle k, 1\rangle \\
& -n \mu\left(1-\frac{1}{\sigma}\right)\langle k, 1\rangle \frac{1}{\sigma}\langle k, 1\rangle \\
& -\operatorname{vol}(\Omega) \mu\left(1-\frac{1}{\sigma}\right)\left(\mu\left(1-\frac{1}{\sigma}\right)\right)^{-\sigma}\langle k, 1\rangle^{1-1 / \sigma} \\
& +\operatorname{vol}(\Omega) \frac{\sigma\left[(\mu(1-1 / \sigma))^{-\sigma}\langle k, 1\rangle\right]^{1-1 / \sigma}}{\sigma-1}
\end{aligned}
$$

where $\operatorname{vol}(\Omega):=\int_{\Omega} 1 \mathrm{~d} x$. To simplify $\mu\langle k, 1\rangle^{1-1 / \sigma}$ (a nonzero factor) in equation (25), we obtain

$$
\begin{aligned}
\rho= & (A-n)\left(1-\frac{1}{\sigma}\right)-\operatorname{vol}(\Omega)\left(1-\frac{1}{\sigma}\right)\left(\mu\left(1-\frac{1}{\sigma}\right)\right)^{-\sigma} \\
& +\operatorname{vol}(\Omega)\left(\mu\left(1-\frac{1}{\sigma}\right)\right)^{-\sigma},
\end{aligned}
$$

which reads

$$
\mu=\frac{\sigma}{\sigma-1}\left(\frac{\sigma \rho-(A-n)(\sigma-1)}{\operatorname{vol}(\Omega)}\right)^{-1 / \sigma} .
$$

Then, we obtain an explicit solution of HJB equation (19), which is of the form

$$
v(k)=\frac{\sigma}{\sigma-1}\left(\frac{\sigma \rho-(A-n)(\sigma-1)}{\operatorname{vol}(\Omega)}\right)^{-1 / \sigma}\langle k, 1\rangle^{1-1 / \sigma} .
$$

On the contrary, the trajectory $k(\cdot)$ related to a control $c(\cdot)$ in set (15) is the solution of the following system:

$$
\left\{\begin{array}{l}
\dot{k}(t)=G k(t)+A k(t)-n k(t)-c(t), \\
k(0)=k_{0},
\end{array}\right.
$$

where $\dot{k}(t):=\partial k(t) / \partial t$. At every time $t$ and point $x$, we observe that $k(\cdot)$ remains below:

$$
\widehat{k}(t)=e^{(A-n) t} e^{G t} \widehat{k}_{0},
$$

where $\widehat{k}(t)$ is the solution of the following system:

$$
\left\{\begin{array}{l}
\dot{\hat{k}}(t)=G \widehat{k}(t)+A \widehat{k}(t)-n \widehat{k}(t), \\
\widehat{k}(0)=\widehat{k}_{0}
\end{array}\right.
$$

Then, we have $\langle\widehat{k}(t), 1\rangle \geq\langle k(t), 1\rangle$ for all $t>0$. Since $\langle\widehat{k}(t), 1\rangle=\left\langle e^{(A-n) t} e^{G t} \widehat{k}_{0}, 1\right\rangle=\left\langle e^{(A-n) t} \widehat{k}_{0}, e^{G t} 1\right\rangle=e^{(A-n) t}\left\langle\widehat{k}_{0}, 1\right\rangle$,

we have a bounded utility:

$$
\begin{aligned}
\left|e^{-\rho t} v(k(t))\right| & =e^{-\rho t} \mu\langle k(t), 1\rangle^{1-1 / \sigma} \\
& \leq e^{-\rho t} \mu\langle\widehat{k}(t), 1\rangle^{1-1 / \sigma} \\
& \leq e^{-\rho t} e^{(A-n)\left(1-\frac{1}{\sigma}\right) t} \mu\left\langle\widehat{k}_{0}, 1\right\rangle^{1-1 / \sigma} .
\end{aligned}
$$

Thus, the bounded utility $\left|e^{-\rho t} v(k(t))\right| \longrightarrow 0$ if

$$
(A-n)\left(1-\frac{1}{\sigma}\right)-\rho<0 .
$$

Inequality (34) yields

$$
n>A+\frac{\sigma \rho}{1-\sigma},
$$

and otherwise, $\left|e^{-\rho t} v(k(t))\right| \longrightarrow+\infty$.

Remark 2. In Proposition 1, condition (20) is applied to guarantee the finiteness of the objective function. Indeed, we also obtain the finiteness of the value function with condition (20) because of explicit expression (21). In the following proposition, we illustrate that the feedback control is admissible and prove that the solution of HJB equation (19) obtained in Proposition 1 is the value function. Furthermore, the optimal control of problem (18) is derived by employing the value function.

Proposition 2. Assume that the hypotheses of Proposition 1 are satisfied. Then, the feedback control $c=(\mu(1-1 / \sigma))^{-\sigma}\langle k, 1\rangle 1$ is the unique optimal control of system (13) and the value function of system (13) computed at $k_{0}$ is

$$
v\left(k_{0}\right)=\frac{\sigma}{\sigma-1}\left(\frac{\sigma \rho-(A-n)(\sigma-1)}{\operatorname{vol}(\Omega)}\right)^{-1 / \sigma}\left\langle k_{0}, 1\right\rangle^{1-1 / \sigma}
$$

Proof. The feedback control linked to the solution of HJB equation (19) is

$$
\left\{\begin{array}{l}
\Phi: M \longrightarrow L^{2}(\Omega), \\
\Phi(k) \in \operatorname{argmax}_{c \in L^{2}(\Omega)}\left\{-\mu\left(1-\frac{1}{\sigma}\right)\langle k, 1\rangle-\frac{1}{\sigma}\langle c, 1\rangle+\langle 1, U(c)\rangle\right\} \\
=\left(\mu\left(1-\frac{1}{\sigma}\right)\right)^{-\sigma}\langle k, 1\rangle 1=\frac{\sigma \rho-(A-n)(\sigma-1)}{\operatorname{vol}(\Omega)}\langle k, 1\rangle 1 .
\end{array}\right.
$$

Choosing $k_{0} \in L^{2}(\Omega)$ and replacing $c(t)$ by the feedback control $\Phi(k(t))$ in system (29), we obtain 


$$
\left\{\begin{array}{l}
\dot{k}(t)=G k(t)+A k(t)-n k(t)-\frac{\sigma \rho-(A-n)(\sigma-1)}{\operatorname{vol}(\Omega)}\langle k, 1\rangle 1, \\
k(0)=k_{0} .
\end{array}\right.
$$

According to the definition of mild solution in Bensoussan et al. [32], we have a mild solution of system (38), which is given by

$$
k_{\Phi}(t)=e^{\widetilde{G} t} k_{0}-\int_{0}^{t} e^{\widetilde{G}((t-s)}\left(\frac{\sigma \rho-(A-n)(\sigma-1)}{\operatorname{vol}(\Omega)}\left\langle k_{\Phi}(s), 1\right\rangle 1\right) \mathrm{d} s,
$$

where $\widetilde{G}:=G+A-n$ is the identity operator.
Note that, due to examples in [32], we know that equation (39) has a unique solution even if it is not an explicit form. We denote the unique solution of equation (39) by $k_{\Phi}^{*}(t)$, which is the related trajectory driven by the feedback control $c^{*}$ with $c^{*}(t):=\Phi\left(k_{\Phi}^{*}(t)\right)$ for all $t>0$. Under the hypothesis of positive $k_{\Phi}^{*}(t)(x), c^{*}(t)$ is positive and admissible.

In general, we know that $c^{*}(\cdot)$ is the optimal control if we obtain

$$
J\left(k_{0}, c^{*}(\cdot)\right) \geq J\left(k_{0}, \bar{c}(\cdot)\right)
$$

In equation $(40), \bar{c}(\cdot)$ denotes any other admissible control of (15). Supposing that $\bar{k}(\cdot)$ is the trajectory linked to the control $\bar{c}(\cdot)$ and considering function $w(t, k): R \times L^{2}(\Omega) \longrightarrow R$ with $w(t, k):=e^{-\rho t} v(k)$, we have

$$
\begin{aligned}
v\left(k_{0}\right)-w(T, \bar{k}(T)) & =w(t, \bar{k}(0))-w(T, \bar{k}(T))=-\int_{0}^{T} \frac{\mathrm{d}}{\mathrm{d} t} w(t, \bar{k}(t)) \mathrm{d} t \\
& =\int_{0}^{T} e^{-\rho t}[\rho v(\bar{k}(t))-\langle G \bar{k}(t)+(A-n) \bar{k}(t)-\bar{c}(t), \nabla v(\bar{k}(t))\rangle] \mathrm{d} t .
\end{aligned}
$$

Regularizing properties of the heat semigroup, we know that $\bar{k}(t) \in D(G)$ for any $t>0$. Using inequality (33), we know that $w(T, \bar{k}(T)) \longrightarrow 0, T \longrightarrow \infty$. Thus, we obtain

$$
v\left(k_{0}\right)=\int_{0}^{+\infty} e^{-\rho t}[\rho v(\bar{k}(t))-\langle(A-n) \bar{k}(t)-\bar{c}(t), \nabla v(\bar{k}(t))\rangle-\langle\bar{k}(t), G \nabla v(\bar{k}(t))\rangle] \mathrm{d} t .
$$

Considering the solution $v(k)$ of equation (19), we have

$$
\begin{aligned}
& v\left(k_{0}\right)-J\left(k_{0}, \bar{c}(\cdot)\right) \\
& =\int_{0}^{+\infty} e^{-\rho t}[\rho v(\bar{k}(t))-\langle A \bar{k}(t), \nabla v(\bar{k}(t))\rangle+\langle n \bar{k}(t), \nabla v(\bar{k}(t))\rangle-\langle\bar{k}(t), G \nabla v(\bar{k}(t))\rangle+\langle\bar{c}(t), \nabla v(\bar{k}(t))\rangle-\langle 1, U(\bar{c}(t))\rangle] \mathrm{d} t \\
& =\int_{0}^{+\infty} e^{-\rho t}\left[\sup _{c \in L^{2}\left(T: R^{+}\right)}\{\langle 1, U(c)\rangle-\langle c, \nabla v(\bar{k}(t))\rangle\}-(\langle 1, U(\bar{c}(t))\rangle-\langle\bar{c}(t), \nabla v(\bar{k}(t))\rangle)\right] \mathrm{d} t \geq 0,
\end{aligned}
$$

$$
J\left(k_{0}, \bar{c}(\cdot)\right) \leq J\left(k_{0}, c^{*}(\cdot)\right) .
$$

which results in

$$
v\left(k_{0}\right)-J\left(k_{0}, \bar{c}(\cdot)\right) \geq 0 .
$$

Expression (44) implies that there exists

$$
v\left(k_{0}\right)-J\left(k_{0}, c^{*}(\cdot)\right)=0,
$$

where $c^{*}(\cdot)$ is a control defined with the feedback in (37). Therefore, for all admissible control $\bar{c}(\cdot)$, we obtain

$$
v\left(k_{0}\right)-J\left(k_{0}, \bar{c}(\cdot)\right) \geq v\left(k_{0}\right)-J\left(k_{0}, c^{*}(\cdot)\right),
$$

which leads to
Inequality (47) shows that $c^{*}(\cdot)$ is the optimal control.

\section{Convergence Analysis of Capital Distribution}

In the long run, the willingness of the planner induces the spatio-temporal dynamics to give a certain consumption which equalizes the capital level in different locations. Thus, the spatio-temporal dynamics eliminates the initial inequalities in capital endowments. This result on decreasing returns plays a key role in the traditional theory of 
convergence. In this section, we discuss the uniform convergence of the detrended capital distribution over space by applying the explicit solution of system (29).

Theorem 1. Assume that the hypotheses of Proposition 1 are satisfied. Then, there exists a closed-form solution of system (29), which is given by

$$
k(t)=k_{0} e^{(G+\sigma A-\sigma n-\sigma \rho) t},
$$

where $k_{0}=c_{0} / \sigma \rho+(A-n)(1-\sigma)$.

The optimal consumption dynamics is an elementary factor of the dynamics programming method, which is used to maximize the utility function and obtain the behavior of optimal capital distribution. Thus, we apply the optimal consumption to complete proof of Theorem 1.

Proof. According to the feedback control $c=$ $(\mu(1-1 / \sigma))^{-\sigma}\langle k, 1\rangle 1$ obtained in Proposition 3.2, we have

$$
\begin{aligned}
\langle c, 1\rangle & =\left\langle\left(\mu\left(1-\frac{1}{\sigma}\right)\right)^{-\sigma}\langle k, 1\rangle 1,1\right\rangle \\
& =\left(\mu\left(1-\frac{1}{\sigma}\right)\right)-\sigma\langle k, 1\rangle\langle 1,1\rangle .
\end{aligned}
$$

By calculation, we get

$$
\int_{\Omega} c(t) \mathrm{d} x=\int_{\Omega}[\sigma \rho+(A-n)(1-\sigma)] k(t) \mathrm{d} x .
$$

Then, we have

$$
c(t)=[\sigma \rho+(A-n)(1-\sigma)] k(t) .
$$

Plugging equation (51) into system (29) and using equation (39), we get the explicit solution of system (29), which is

$$
\begin{aligned}
k(t)= & e^{G t} e^{A t} e^{-n t} k_{0}-\int_{0}^{t} e^{(t-s) G} e^{(t-s) A} e^{-(t-s) n} c(s) \mathrm{d} s \\
= & e^{G t} e^{A t} e^{-n t} k_{0}-\frac{c_{0}}{\sigma \rho+(A-n)(1-\sigma)} e^{(G+A-n) t} \\
& +\frac{c_{0}}{\sigma \rho+(A-n)(1-\sigma)} e^{G t+\sigma(A-n-\rho) t} \\
= & k_{0} e^{(G+\sigma A-\sigma n-\sigma \rho) t},
\end{aligned}
$$

where $k_{0}=c_{0} / \sigma \rho+(A-n)(1-\sigma)$. Equation (52) describes the time behavior of per capita capital in system (29).

According to the works in [10], we know that the behavior of spatial capital distribution implies composite transitional dynamics because of the second order term $\triangle_{x} k(x, t)$ in system (7). Precisely, the aggregate level of capital distribution in the spatial model is equivalent to that in corresponding form of the one-dimensional model. In the following theorems, we discuss the characterization of aggregate capital with the optimal control obtained in Proposition 2.
Theorem 2. Under the assumptions of Proposition 1, there exists per aggregate capital $K(t)$ with the optimal control, which is given by

$$
K(t)=e^{\beta t} K(0),
$$

where $\beta:=\sigma(A-n-\rho)$ and $K(0)=\int_{\Omega} k_{0}(x) d x$.

Proof. Using closed-form solution (48), we obtain

$$
\begin{aligned}
K(t) & =\langle k(t), 1\rangle=\left\langle k_{0} e^{(G+\sigma A-\sigma n-\sigma \rho) t}, 1\right\rangle \\
& =e^{\sigma(A-n-\rho) t}\left\langle k(0), e^{G t} 1\right\rangle \\
& =e^{\sigma(A-n-\rho) t}\langle k(0), 1\rangle \\
& =e^{\sigma(A-n-\rho) t} \int_{\Omega} k(0) \mathrm{d} x \\
& =e^{\sigma(A-n-\rho) t} K(0) \\
& =e^{\beta t} K(0),
\end{aligned}
$$

where $\beta:=\sigma(A-n-\rho)$ and $K(0)=\int_{\Omega} k_{0}(x) \mathrm{d} x$.

Remark 3. According to the evolution of optimal aggregate capital stock in the spatial growth model, we find that the aggregate capital grows with an explicit growth rate $\beta$ from $K(0)$. By (51) and (53), we obtain the relationship between the optimal control $c^{*}(\cdot)$ and the explicit growth rate $\beta$, which means that, for all individuals, the consumption level chosen by planners is independent of their location and generation. In the following theorem, we analyze the induced optimal spatio-temporal dynamics of detrended capital when the bounded population growth rate satisfies certain conditions.

Theorem 3. Suppose that the hypotheses of Proposition 1 hold. Then, for a real number $r_{1}$, if

$$
n<A+\frac{\rho \sigma}{1-\sigma}+\frac{\sigma r_{1}^{2}}{\sigma-1}
$$

the detrended capital

$$
k_{D}(x, t):=e^{-\beta t} k(x, t),
$$

converges uniformly to a constant $K(0) / \operatorname{vol}(\Omega)$ when $t$ tends to infinity.

In Theorem 3, the optimal spatial dynamics of capital, which is induced by the optimal consumption obtained in Proposition 2, guarantees the asymptotic behavior of detrended capital. Inequalities in initial endowments are eliminated by the spatial dynamics of capital to equalize the level of capital across locations in the long run. This fact is due to the explicit expression of the capital in Theorem 1 and a threshold value of the population growth rate $\left(n<\bar{n}=A+\rho \sigma / 1-\sigma+\sigma r_{1}^{2} / \sigma-1\right)$. In the sequel, we provide the proof of Theorem 3 by employing corresponding form of Fourier series. 
Proof. Firstly, we write $k_{D}(x, t)$ by using Fourier series. For $j \in Z$, we denote the eigenfunction of $\Delta_{x}$ by a regular function $\phi_{j}: \Omega \longrightarrow R$, such that

$$
\triangle_{x} \phi_{j}(x)=-r_{j}^{2} \phi_{j}(x) .
$$

In equation (57), $r_{j}$ is a real number eigenvalue in the set of eigenvalues which is discrete as form of the sequence $0=$ $r_{0}<r_{1}<r_{2}<\cdots<r_{j}<\cdots[35]$. Therefore, for fixed $x$ and considering $\varphi(x, t)=e^{G t} \phi_{j}(x)$, we obtain

$\frac{\mathrm{d} \varphi(x, t)}{\mathrm{d} t}=e^{G t} G \phi_{j}(x)=e^{G t} \triangle_{x} \phi_{j}(x)=-r_{j}^{2} e^{G t} \phi_{j}(x)=-r_{j}^{2} \varphi(x, t)$.

Equation (58) yields

$$
(x, t)=e^{-r_{j}^{2} t} \varphi(x, 0),
$$

which implies

$$
e^{G t} \phi_{j}(x)=e^{-r_{j}^{2} t} \phi_{j}(x)
$$

Then, we obtain

$$
e^{A t} e^{G t} \phi_{j}(x)=e^{\left(A-r_{j}^{2}\right) t} \phi_{j}(x) .
$$

In order to determinate the Fourier coefficients, using equation (48), we take the scalar product with $\phi_{j}$ for all $j$ and obtain

$$
\begin{aligned}
\left\langle k(t), \phi_{j}\right\rangle & =\left\langle k_{0}, e^{(G+\sigma A-\sigma n-\sigma \rho) t} \phi_{j}\right\rangle \\
& =e^{\left(\sigma A-\sigma n-r_{j}^{2}-\sigma \rho\right) t}\left\langle k_{0}, \phi_{j}\right\rangle, \quad j \neq 0 .
\end{aligned}
$$

Particularly, if $j=0$, we get

$$
\left\langle k_{D}(t), \phi_{0}\right\rangle=e^{-\sigma(A-n-\rho) t}\left\langle k(t), \phi_{0}\right\rangle=\left\langle k_{0}, \phi_{0}\right\rangle=\frac{K(0)}{\sqrt{\operatorname{vol}(\Omega)}},
$$

where the unique normalized function is

$$
\phi_{0}=\frac{1}{\sqrt{\operatorname{vol}(\Omega)}} 1 \text {. }
$$

Applying Fourier series to write functions on $\Omega$, we have

$$
\left|k-\sum_{j \in z} \phi_{j}\left\langle k, \phi_{j}\right\rangle\right|_{L^{2}(\Omega)}=0,
$$

for every function $k \in L^{2}(\Omega)$ [36]. In this case, we express $k_{D}(t)$ as

$$
\begin{aligned}
k_{D}(t)(x) & =\sum_{j \in z}\left\langle k_{D}(t), \phi_{j}\right\rangle \phi_{j}(x) \\
& =\sum_{j \in z} e^{-\beta t}\left\langle k_{t}, \phi_{j}\right\rangle \phi_{j}(x) \\
& =\frac{K(0)}{\operatorname{vol}(\Omega)}+\sum_{j \in z, j \neq 0} e^{-\beta t} e^{\left(\sigma A-\sigma n-r_{j}^{2}-\sigma \rho\right) t}\left\langle k_{0}, \phi_{j}\right\rangle \phi_{j}(x) \\
& =\frac{K(0)}{\operatorname{vol}(\Omega)}+\sum_{j \in z, j \neq 0} e^{\left(\sigma A-\sigma n-r_{j}^{2}-\sigma \rho-\beta\right) t}\left\langle k_{0}, \phi_{j}\right\rangle \phi_{j}(x) .
\end{aligned}
$$

Using $|k|_{L^{2}(T)}^{2}=\sum_{j \in z}\left|\left\langle k, \phi_{j}\right\rangle\right|^{2}$ and expression of $k_{D}(t)$ as Fourier series, we have

$$
\begin{aligned}
& \left|k_{D}(t)(x)-\frac{K(0)}{\operatorname{vol}(\Omega)}\right|_{L^{2}(\Omega)}^{2} \\
& =\sup _{x \in \Omega}\left|\sum_{j \in z, j \neq 0} e^{\left(\sigma A-\sigma n-r_{j}^{2}-\sigma \rho-\beta\right) t}\left\langle k_{0}, \phi_{j}\right\rangle \phi_{j}(x)\right|^{2} \\
& \leq\left. e^{-2 \varepsilon t}\left|\sum_{j \in z, j \neq 0} e^{\left(\sigma A-\sigma n-r_{j}^{2}-\sigma \rho-\beta+\varepsilon\right) t}\right|\left\langle k_{0}, \phi_{j}\right\rangle\left|\sup _{x \in \Omega}\right| \phi_{j}(x)\right|^{2} \\
& \leq S^{2} e^{-2 \varepsilon t}\left(\sum_{j \geq 1} e^{2\left(\sigma A-\sigma n-r_{j}^{2}-\sigma \rho-\beta+\varepsilon\right) t}\right)\left(\sum_{j \geq 1}\left|\left\langle k_{0}, \phi_{j}\right\rangle\right|^{2}\right) \\
& =S^{2} e^{-2 \varepsilon t}\left(\sum_{j \geq 1} e^{2\left(\sigma A-\sigma n-r_{j}^{2}-\sigma \rho-\beta+\varepsilon\right) t}\right)\left|k_{0}\right|_{L^{2}}^{2},
\end{aligned}
$$

where $S$ is a positive constant and satisfies $\left|\sup _{x \in \Omega}\right| \phi_{j}(x) \mid \leq S$.

Since

$$
A+\frac{\rho \sigma}{1-\sigma}<n<A+\frac{\rho \sigma}{1-\sigma}+\frac{\sigma r_{1}^{2}}{\sigma-1}
$$

we have

$$
(A-n)\left(1-\frac{1}{\sigma}\right)<\rho<(A-n)\left(1-\frac{1}{\sigma}\right)+r_{1}^{2} .
$$

Considering $\epsilon \in\left(0,(A-n)(\sigma-1)+\sigma r_{1}^{2}-\sigma \rho / \sigma\right)$, we obtain

$$
\begin{aligned}
& \sigma A-\sigma n-r_{j}^{2}-\sigma \rho-\beta+\varepsilon \\
& <\sigma A-\sigma n-r_{1}^{2}-\sigma \rho-\beta+\frac{(A-n)(\sigma-1)+\sigma r_{1}^{2}-\sigma \rho}{\sigma} \\
& =\frac{(A-n)(\sigma-1)-\sigma \rho}{\sigma}<0 .
\end{aligned}
$$

Let $H:=\sum_{j \geq 1} e^{2\left(\sigma A-\sigma n-r_{j}^{2}-\sigma \rho-\beta+\varepsilon\right) t}$. Then, we know $H<+\infty$ due to condition (70). Therefore, we rewrite $(67)$ as

$$
\left|k_{D}(t)(x)-\frac{K(0)}{\operatorname{vol}(\Omega)}\right|_{L^{2}(\Omega)}^{2} \leq e^{-2 \varepsilon t}\left(S^{2} H\left|k_{0}\right|_{L^{2}}^{2}\right) \longrightarrow 0,
$$

which completes the proof.

\section{Numerical Simulations}

In the spatial economic model, the adjustment of optimal trajectory is not instantaneous when the spatial capital flows from rich regions to poor regions. In the rich area, the flow of capital brings a temporary production agglomeration, while a depressed condition appears in the poor area. After that, the spatial capital in the rich location moves to the poor location, and then, the optimal path is formed. In this 


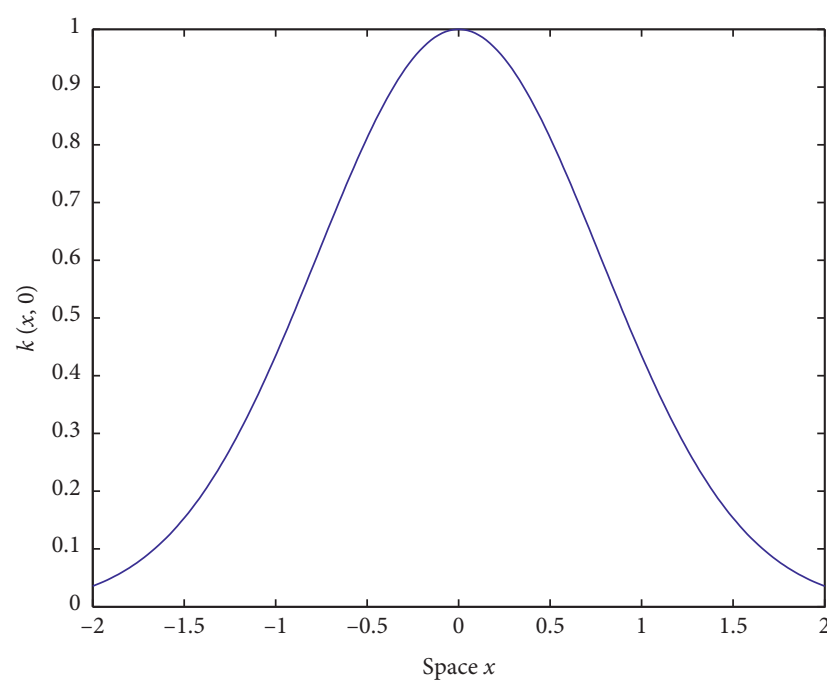

(a)

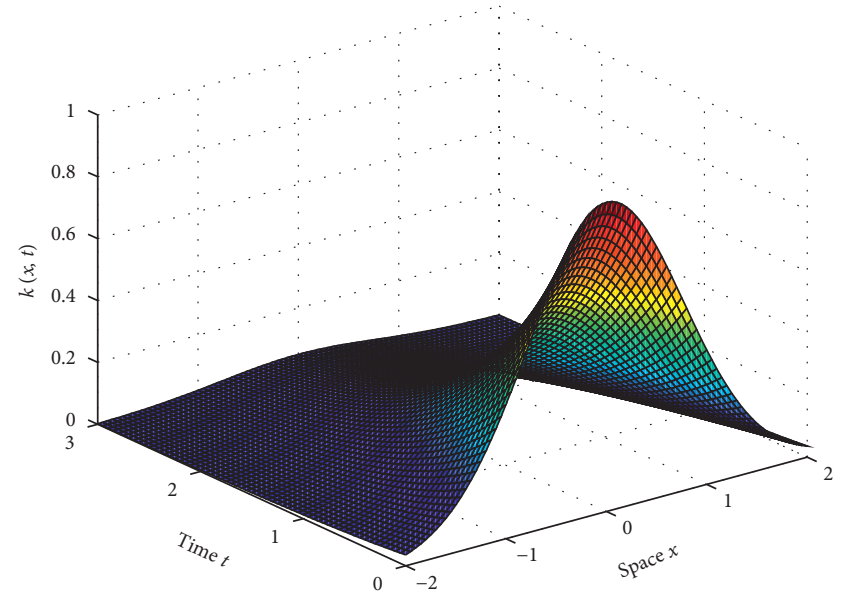

(b)

Figure 1: Evolution of the spatial capital distribution corresponding to $A=0.1, n=0.03, \rho=0.05, \sigma=3$, and $r_{1}=0.1$.

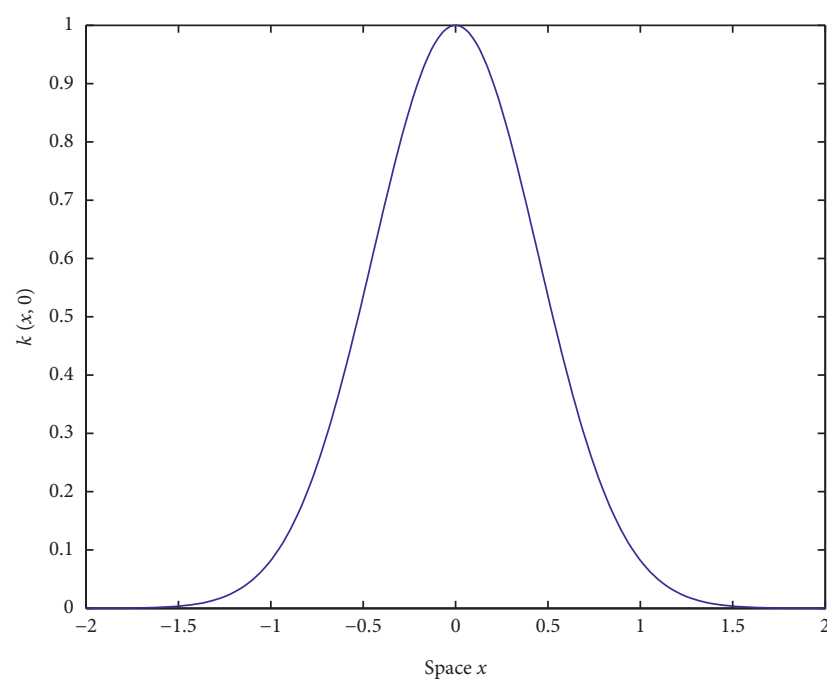

(a)

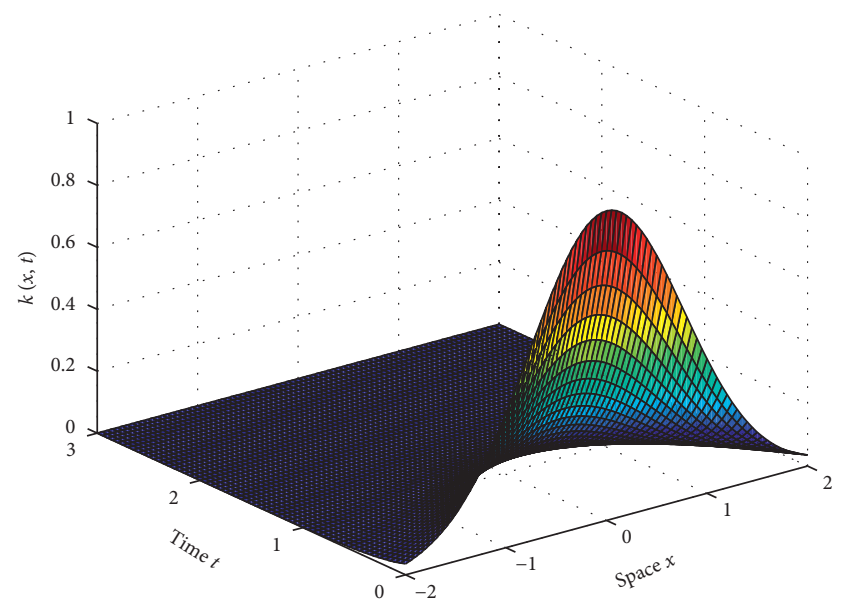

(b)

FIgURe 2: Evolution of the spatial capital distribution in the case $n(1-\sigma)=A(1-\sigma)+\rho \sigma-\sigma r_{1}^{2}$.

section, we consider different values of relevant parameters to show the formation of the optimal path and illustrate results of the spatial capital distribution with exponential decays.

We simulate two cases of initial capital distribution to illustrate approximate profiles of the physical capital across space and time when some values of relevant parameters are given. As shown in Figure 1, the optimal trajectory of spatial capital distribution corresponds to $A=$ $0.1, n=0.03, \rho=0.05, \sigma=3$, and $r_{1}=0.1$, which satisfy conditions (20) and (55). In the initial stage, we observe that the spatial capital distribution is highly clustered in the center section of space interval, while it is very dispersed elsewhere. This case is consistent with the optimal spatio-temporal dynamics of the detrended capital since we know that, as a sum of different terms, the second term on the right of equation (66) is invisible because of the exponential factor $e^{\left(\sigma A-\sigma n-r_{j}^{2}-\sigma \rho-\beta\right) t}$ with condition (68). Precisely, the limit of detrended capital $k_{D}(t)(x)$ is the term $K(0) / \operatorname{vol}(\Omega)$ when $t \longrightarrow \infty$. On the contrary, if we change values of the relevant parameters to satisfy $n(1-\sigma)=A(1-\sigma)+\rho \sigma-\sigma r_{1}^{2}$, Figure 2 shows the corresponding profiles of the initial capital and spatial capital distribution. In this case, the detrended capital is not convergent to a spatially homogeneous distribution in the long run since the term $\sum_{j \in z, j \neq 0} e^{\left(\sigma A-\sigma n-r_{j}^{2}-\sigma \rho-\beta\right) t}\left\langle k_{0}, \phi_{j}\right\rangle \phi_{j}(x)$ is involved in the limit. 


\section{Conclusions}

In this paper, we have investigated the optimal dynamics of a spatial economic growth model with bounded population growth in a continuous space. Considering the growth model redrafted in the infinite dimensional Hilbert space, we apply the HJB equation and linear production function to identify the unique optimal control and the value function of the growth model. Under assumption of the bounded population growth, the explicit solution of our model is derived in close form by using the optimal control, which satisfies the first-order optimality conditions to show the time behavior of per capita capital. With nonnegative initial capital distribution, we apply the explicit solution and Fourier series to express the detrended capital of our model and then prove the convergence of the detrended capital level across space and time.

\section{Data Availability}

No data were used to support the findings of this study.

\section{Conflicts of Interest}

The author declares that there are no conflicts of interest regarding the publication of this paper.

\section{Acknowledgments}

This work was supported by the Talent Introduction Program Funds of Xihua University (Z201072).

\section{References}

[1] M. Ferrara, L. Guerrini, and M. Sodini, "Nonlinear dynamics in a Solow model with delay and non-convex technology," Applied Mathematics and Computation, vol. 228, pp. 1-12, 2014.

[2] W. A. Brock, A. Xepapadeas, and A. N. Yannacopoulos, "Spatial externalities and agglomeration in a competitive industry," Journal of Economic Dynamics and Control, vol. 42, pp. 143-174, 2014.

[3] S. Anita, V. Capasso, H. Kunze, and D. L. Torre, "Optimal control and long-run dynamics for a spatial economic growth model with physical capitalaccumulation and pollution diffusion," Applied Mathematics Letters, vol. 26, pp. 908-912, 2013.

[4] R. Boucekkine, G. Fabbri, and P. A. Pintus, "Short-run pain, long-run gain: the conditional welfare gains from international financial integration," Economic Theory, vol. 65, no. 2, pp. 329-360, 2018.

[5] C. Camacho, B. Zou, and M. Briani, "On the dynamics of capital accumulation across space," European Journal of Operational Research, vol. 186, no. 2, pp. 451-465, 2008.

[6] M. Beckmann, "A continuous model of transportation," Econometrica, vol. 20, no. 4, pp. 643-660, 1952.

[7] W. Isard and P. Liossatos, Spatial Dynamic and Optimal Space-Time Development, North-Holland Publishing Company, Amsterdam, Netherlands, 1979.

[8] P. Brito, The Dynamics of Growth and Distribution in a Spatially Heterogeneous World, Department of Economics,
ISEG, University of Lisbon, Lisboa, Portugal, Working papers, 2004.

[9] R. Boucekkine, C. Camacho, and B. Zou, "Bridging the gap between growth theory and the new economic geography: the spatial Ramsey model," Macroeconomic Dynamics, vol. 13, no. 1, pp. 20-45, 2009.

[10] R. Boucekkine, C. Camacho, and G. Fabbri, "Spatial dynamics and convergence: the Spatial AK model," Journal of Economic Theory, vol. 148, no. 6, pp. 2719-2736, 2013.

[11] G. Fabbri, "Geographical structure and convergence: a note on geometry in spatial growth models," Journal of Economic Theory, vol. 162, pp. 114-136, 2016.

[12] L. Guerrini, "The Ramsey model with AK technology and a bounded population growth rate," Journal of Macroeconomics, vol. 32, no. 4, pp. 1178-1183, 2010.

[13] D. La Torre, D. Liuzzi, and S. Marsiglio, "Pollution diffusion and abatement activities across space and over time," Mathematical Social Sciences, vol. 78, pp. 48-63, 2015.

[14] T. Kamihigashi and S. Roy, "Dynamic optimization with a nonsmooth, nonconvex technology: the case of a linear objective function," Economic Theory, vol. 29, no. 2, pp. 325-340, 2006.

[15] S. Brianzoni, C. Mammana, and E. Michetti, "Local and global dynamics in a discrete time growth model with nonconcave production function," Discrete Dynamics in Nature and Society, vol. 2012, Article ID 536570, 22 pages, 2012.

[16] M. Capasso, E. Stam, and E. Cefis, "Industrial dynamics and economic geography," Regional Studies, vol. 49, no. 1, pp. 5-9, 2015.

[17] C. Camacho and B. Zou, "The spatial Solow model," Economics Bulletin, vol. 18, pp. 1-11, 2004.

[18] V. Capasso, R. Engbers, and D. La Torre, "On a spatial Solow model with technological diffusion and nonconcave production function," Nonlinear Analysis: Real World Applications, vol. 11, no. 5, pp. 3858-3876, 2010.

[19] W. A. Brock, A. Xepapadeas, and A. N. Yannacopoulos, "Optimal agglomerations in dynamic economics," Journal of Mathematical Economics, vol. 53, pp. 1-15, 2014.

[20] A. Xepapadeas and A. N. Yannacopoulos, "Spatial growth with exogenous saving rates," Journal of Mathematical Economics, vol. 67, pp. 125-137, 2016.

[21] Z. Yue and H. Wenyi, "Spatial dynamics for a generalized Solow growth model," Discrete Dynamics in Nature and Society, vol. 2018, Article ID 6945032, 8 pages, 2018.

[22] P. Mossay, "A theory of rational spatial agglomerations," Regional Science and Urban Economics, vol. 43, no. 2, pp. 385-394, 2013.

[23] T. Mingyu, Y. Zou, and C. Liu, "Research on a grey prediction model of population growth based on a logistic approach," Discrete Dynamics in Nature and Society, vol. 2020, Article ID 2416840, 14 pages, 2020.

[24] S. Marsiglio and M. Tolotti, "Endogenous growth and technological progress with innovation driven by social interactions," Economic Theory, vol. 65, no. 2, pp. 293-328, 2018.

[25] G. Fabbri, S. Faggian, and G. Freni, Spatial Resource Wars: A Two Region Example, Department of Economics CaFoscari University of Venice, Venezia, VE, Italy, 2018.

[26] A. G. Abadi, "Global stability of malaria transmission dynamics model with logistic growth," Discrete Dynamics in Nature and Society, vol. 2018, Article ID 5759834, 12 pages, 2018.

[27] L.-I. Anita, S. Anita, and V. Arnautu, "Global behavior for an age-dependent population model with logistic term and periodic vital rates," Applied Mathematics and Computation, vol. 206, pp. 368-379, 2008. 
[28] P. Brito, "Asymptotic expansion of solutions to the BlackScholes equation arising from American option pricing near the expiry," Journal of Computational and Applied Mathematics, vol. 311, pp. 11-37, 2017.

[29] H. Mi and L. Xu, "Optimal investment with derivatives and pricing in an incomplete market," Journal of Computational and Applied Mathematics, vol. 368, Article ID 112522, 2020.

[30] S. Chatterjee, "Transitional dynamics and the distribution of wealth in a neoclassical growth model," Journal of Public Economics, vol. 54, no. 1, pp. 97-119, 1994.

[31] A. Grigoryan, Heat kernel and analysis on manifolds, American Mathematical Society, Providence, Rhode Island, 2012.

[32] A. Bensoussan, G. D. Prato, M. C. Delfour, and S. K. Mitter, Representation and Control of Infinite Dimensional System, Birkhäuser Boston, Cambridge, MA, USA, 2nd edition, 2007.

[33] J. L. Lions and E. Magenes, Non-Homogeneous Bundary Valie Problems and Applications, Vol. 3, Springer-Verlag, New York, NY, USA, 1972.

[34] X. Li and J. Yong, Optimal Control Theory for Infinite Dimensional Systems, Birkhäuser, Berlin, Germany, 1995.

[35] B. Chow, P. Lu, and L. Ni, "Hamilton's ricci flow," Graduate Studies in Mathematics, vol. 77, 2006.

[36] S. Rudin, Real and Complex Analysis, McGraw-Hill, New York, NY, USA, 1987. 